\title{
SOBREVIDA ESPECÍFICA DE PACIENTES COM CÂNCER DE MAMA NÃO-METASTÁTICO SUBMETIDAS À QUIMIOTERAPIA ADJUVANTE
}

Jane Rocha Duarte Cintra*, Maximiliano Ribeiro Guerra, Maria Teresa Bustamante-Teixeira

Trabalho realizado pelo NATES/Faculdade de Medicina - UFJF - Programa de Pós-graduação em Saúde Brasileira, Juiz de Fora, MG

*Correspondência Instituto Oncológico/ Hospital 9 de Julho Rua Santos Dumont, 56 CEP $36035-370$

Juiz de Fora - MG janerdc@terra.com.br

\section{RESUMO}

OBjEtivo. Analisar a sobrevida específica de cinco anos em mulheres com câncer de mama invasivo não-metastático, e que foram submetidas à cirurgia com intenção curativa e quimioterapia adjuvante.

Métodos. Foram selecionadas 428 pacientes a partir de todos os serviços de oncologia da cidade de Juiz de Fora (MG) com diagnóstico da doença efetuado entre janeiro de 1998 e dezembro de 2000. A data do diagnóstico histopatológico da doença foi considerada como ińíio do tempo de sobrevida e a data do óbito pela doença foi considerada como o evento adverso. Foram censuradas as mulheres que permaneceram vivas até dezembro de 2005, data final do seguimento. Para aquelas que interromperam o seguimento, a data da censura foi referente ao último acompanhamento no registro médico. As curvas de sobrevida foram estimadas pelo método de Kaplan-Meier e as diferenças observadas foram avaliadas pelo teste de log-rank. Resultados. A idade média das pacientes foi de 51,2 anos, sendo a maioria das pacientes $(72,6 \%)$ da raça branca. Os estadios clínicos predominantes foram II (47,4\%) e III (38,6\%). A função de sobrevida espećíica para o câncer de mama, no período de cinco anos, foi de $82 \%$. Entre as principais características associadas com uma melhor sobrevida na população de estudo destacaram-se: pré-menopausa $(p=0,02)$, raça branca $(p=0,08)$, tamanho do tumor $\leq 2,0 \mathrm{~cm}(p=0,05)$, ausência de comprometimento linfonodal $(p=0,000)$, estádios mais precoces da doença $(p=0,000)$, realização de ciclos completos de quimioterapia adjuvante $(p=0,03)$ e uso de hormonioterapia $(p=0,05)$.

Conclusáo. Esta pesquisa possibilitou uma melhor caracterização do perfil e da sobrevida de pacientes com câncer de mama que utilizaram terapia quimioterápica adjuvante. Foi constatada a necessidade de ampliação, por parte dos órgãos competentes do setor de saúde local, do rastreamento do câncer de mama, especialmente para o grupo de mulheres considerado como de maior risco, bem como a garantia de tratamento oportuno para todos os casos diagnosticados, o que poderá influenciar positivamente o prognóstico da doença.

UnIteRMos: Câncer da mama. Quimioterapia. Fatores prognósticos. Análise de sobrevivência.

\section{INTRODUÇÃO}

câncer da mama é a neoplasia de maior incidência entre as mulheres no mundo, com cerca de um milhão de casos novos a cada ano ${ }^{1,2,3}$, sendo o segundo câncer mais comum em ambos os sexos, após o de pulmão $0^{3,4}$, e representando a principal causa de morte por neoplasias em mulheres ${ }^{1,3}$. Em homens, esse câncer constitui apenas menos de I\% dos casos novos, resultando em uma proporção mulher/ homem de aproximadamente 100: $1^{5}$.

As mais altas taxas de incidências do câncer de mama são verificadas na América do Norte, Austrália e Nova Zelândia e Europa Ocidental, enquanto na maior parte dos continentes africano e asiático e na América Central, observa-se uma incidência reduzida'1,3,6,7.

A partir das estimativas de incidência e de mortalidade, verifica-se que a América do Sul se encontra em uma posição intermediária no ranking desta doença, com taxa de incidência de 46,0/100.000 e taxa de mortalidade de 15,10/100.000, ambas ajustadas pela população mundial, sendo o Brasil o terceiro país, com taxas de incidência e de mortalidade de 46,0/100.00 e 14,10/100.000, respectivamente ${ }^{7}$.
No Brasil, foram estimadas para 2006 uma taxa de incidência de 52,0 por 100.000 mulheres (48.930 casos novos) e uma taxa de mortalidade de 10,4 por 100.000 mulheres (9335 óbitos), de acordo com dados do Ministério de Saúde. Na Região Sudeste, o câncer de mama é o mais incidente entre as mulheres, com um risco estimado de 7I casos por 100 mil$^{8}$.

A taxa de incidência de câncer de mama varia de acordo com a faixa etária, exibindo um aumento contínuo com a progressão da idade, aumento este que, em muitos países, é freqüentemente seguido de uma redução após a menopausa',2,9.

O estadiamento representa um importante instrumento de avaliação do prognóstico em mulheres com câncer de mama, considerando, em conjunto, as características anatomopatológicas do tumor primário, o comprometimento linfonodal regional e as metástases à distância $^{10}$. No Brasil, predomina o estádio III no momento do diagnóstico, sendo que, recentemente, tem sido observada uma diminuição de casos com o estádio IV e um aumento daqueles com o estadio II $^{\prime !}$. 
CINTRA JRD ET AL.

Os tipos histológicos predominantes dos carcinomas de mama são o tipo ductal e o tipo lobular (in situ ou infiltrante), sendo que o carcinoma ductal infiltrante corresponde a, aproximadamente, 75\% dos casos".

Tendo em vista a importância desta patologia no âmbito da saúde coletiva mundial, bem como a carência de estudos sobre as características do câncer de mama na mulher brasileira, torna-se necessário ampliar os conhecimentos acerca da evolução dessa doença em nosso país, a fim de que possam ser estabelecidas políticas de saúde que propiciem abordagens terapêuticas mais adequadas. $\mathrm{O}$ objetivo deste estudo foi avaliar a sobrevida específica em cinco anos de mulheres com câncer de mama invasivo não-metastático que foram submetidas à cirurgia com intenção curativa seguida de quimioterapia adjuvante, com terapêutica realizada em município da Região Sudeste brasileira.

\section{Métodos}

\section{População de estudo}

A população deste estudo foi composta por uma coorte retrospectiva de base hospitalar formada por 428 mulheres com diagnóstico de câncer de mama invasivo não- metastático, efetuado entre janeiro de 1998 e dezembro de 2000, e que foram submetidas a tratamento cirúrgico com intenção curativa, seguido de quimioterapia adjuvante, sendo a cirurgia e/ou terapia complementar realizada na cidade de Juiz de Fora (MG). Foram excluídas do estudo as pacientes que apenas receberam tratamento paliativo e aquelas que não realizaram terapia adjuvante quimioterápica.

\section{Coleta de informação}

Esta pesquisa foi efetuada a partir de banco de dados de tese de doutorado do Programa de Pós-Graduação em Saúde Coletiva do Instituto de Medicina Social da Universidade do Estado do Rio de Janeiro (IMS/UERJ) $)^{12}$. A construção de tal banco se baseou em informações dos registros e prontuários de todos os serviços de saúde que prestam atendimento em oncologia na referida cidade, por meio de busca ativa nos arquivos médicos, com a utilização de ficha de coleta padronizada. A data limite de seguimento dos casos foi até o final do ano de 2005, período que compreendeu cinco anos após o diagnóstico da última paciente incluída no estudo. O acompanhamento foi efetuado através de retorno aos prontuários médicos, seguido de busca no banco do Sistema de Informação sobre Mortalidade (SIM). Para as pacientes que interromperam o seguimento, foi tentado contato telefônico e, posteriormente, consulta de situação cadastral no Cadastro de Pessoas Físicas (CPF) apenas para obtenção de informação sobre o estado vital.

Inicialmente, foram identificados 868 casos de câncer de mama feminino, os quais representaram todos os casos assistidos no município e diagnosticados no período estabelecido para o estudo. Foram excluídos os casos de carcinoma ductal e lobular in situ $(n=13)$, e aqueles submetidos à cirurgia apenas com finalidade diagnóstica $(n=|| \mid)$. A seguir, foram excluídos $3 \mid$ casos com doença metastática ao diagnóstico. Após esta etapa, foram identificados 713 casos cirúrgicos com doença invasiva (82,1\% da população inicialmente identificada), inicialmente distribuídos nos estadios I, II e III, de acordo com a Classificação TNM da União Internacional Contra o Câncer (UICC) ${ }^{13}$. Passou-se, então, para a etapa de seleção das pacientes que receberam tratamento quimioterápico adjuvante isolado ou associado à radioterapia e/ou à hormonioterapia. Assim, foram excluídos os casos que receberam apenas radioterapia ou hormonioterapia isolados ( $n=99$ casos) ou associados ( $n=133$ casos), e aqueles com ausência de tratamento complementar $(n=12)$. Finalmente, em função dos critérios de elegibilidade adotados para este estudo, foram excluídos os casos que receberam apenas quimioterapia primária isolada $(n=19)$ e aqueles submetidos à quimioterapia paliativa em decorrência de doença metastática diagnosticada através dos exames de estadiamento realizados somente após a cirurgia $(n=22)$.

\section{Variáveis do estudo}

Para este estudo, foram analisadas a data do diagnóstico; variáveis sócio-demográficas: idade ao diagnóstico (em anos), categorizada em: 25-39, 40-49, 50-69 e maior ou igual a 70, e dicotomizada em menor ou igual a 50, e maior que 50 - ponto de corte validado como marcador para status menopausal ${ }^{14}$, cor da pele, município de residência e estado vital; variáveis relativas aos serviços de saúde: natureza do serviço de oncologia (público: conveniado com o Sistema Único de Saúde - SUS, ou privado) e presença de plano privado de saúde; e características tumorais: tamanho tumoral (categorizado em: $\leq 2,0 \mathrm{~cm}$ e $>2,0 \mathrm{~cm}$ ), tipo histológico (ductal infiltrante, lobular infiltrante, outras variantes), comprometimento linfonodal, número de linfonodos isolados (nenhum, I-9 e $\geq 10$ ), estadiamento, presença de metástases, presença de receptores hormonais. Entre os aspectos relativos à quimioterapia adjuvante, foram analisados: data de início; tipo de esquema realizado, categorizado em regimes que utilizavam ciclofosfamida, methotrexate e fluorouracil (CMF) e regimes com antracíclicos (FAC, FEC ou AC); número de ciclos realizados, categorizado em esquema completo ( $\geq 6$ ciclos para CMF, FAC ou FEC, e $\geq 4$ ciclos para AC) e incompleto. No tocante às outras variáveis relacionadas ao tratamento, foram avaliados: tipo de cirurgia (conservadora ou radical); uso de radioterapia (data de início); e uso de hormonioterapia (datas de início e de término).

No que se refere aos casos com acompanhamento inferior a 60 meses, considerou-se que o seguimento era completo apenas para os casos que foram a óbito, sendo os demais considerados como seguimento incompleto.

\section{Análise dos dados}

As diferenças na distribuição das variáveis do estudo foram avaliadas pelo teste Qui quadrado $\left(\chi^{2}\right)$, considerando-se estatisticamente significativas aquelas com p-valor menor que 0,05.

Foi analisada a sobrevida específica em cinco anos, considerandose a data do diagnóstico histopatológico da doença como o início do tempo de sobrevida. Foram considerados como evento adverso (falha) os óbitos (data do óbito) em decorrência do câncer de mama, ou em conseqüência do seu tratamento.

Foram censuradas as pacientes que permaneceram vivas até a data final de seguimento (dezembro de 2005) e aquelas com perda do seguimento, considerando-se, para estas últimas, a data referente ao último acompanhamento registrado no prontuário médico como a data 
da censura. Para oito casos de óbito por causa não relacionada ao câncer de mama, a data da censura considerada foi a do óbito.

As probabilidades de sobrevida foram calculadas de acordo com o método proposto por Kaplan-Meier ${ }^{15}$, com estimativas de curvas de sobrevida, agrupando-se as pacientes segundo as variáveis selecionadas para o estudo. O teste de log-rank foi empregado para a comparação das funções de sobrevida para cada variável.

Toda a análise foi feita utilizando-se os programas EPI INFO 2002. para a entrada e análise descritiva dos dados, e STATA, versão 7.0, para a análise de sobrevida.

A realização do presente estudo foi autorizada pelo Comitê de Ética e Pesquisa da Universidade Federal de Juiz de Fora - Parecer n . 293/3006.

\section{Resultados}

A distribuição das principais variáveis do estudo é apresentada na Tabela I. Foi identificado que 232 pacientes (54,2\%) eram provenientes de cidades vizinhas. A idade média ao diagnóstico foi de 51,2 anos (amplitude 25 a $8 \mathrm{I}$ anos), com mediana de 50 anos, sendo que a maioria das pacientes $(n=299$ - 72,6\%) era da raça branca. Cerca de 48, 1 \% dos casos teve seu diagnóstico histopatológico efetuado no momento da cirurgia. Quanto ao subtipo histológico, o predominante foi o carcinoma ductal infiltrante $(87,4 \%)$, sendo o restante representado pelo lobular infiltrante $(6,3 \%)$ e outras variantes histológicas (6,3\%). Foram evidenciados 274 pacientes (64\%) com tumor maior que $2,0 \mathrm{~cm}$. Entre as mulheres que tinham tumor maior que $2,0 \mathrm{~cm}, 71,1 \%$ também apresentavam comprometimento linfonodal. Foi verificado comprometimento linfonodal em 256 casos $(59,8 \%)$. A maioria das pacientes $(n=366-85,5 \%)$ apresentou mais de 10 linfonodos ressecados.

Os estadios clínico-patológicos II e III foram os predominantes (86\%), sendo que, entre as pacientes com estadiamento III $(n=165)$, $27,9 \%$ faleceram $(n=46)$. No tocante ao estado menopausal, observaram-se maiores percentuais de casos com estadios I e II (54,9\% e $57,1 \%$, respectivamente) e menor percentual de casos com estadio III $(40,6 \%)$ em mulheres na pré-menopausa, em relação às mulheres na pós-menopausa $(p=0,0 \mathrm{I})$. A primeira metástase mais freqüentemente observada foi a óssea (44,3\%), seguida pela pleuro-pulmonar (30,6\%).

Todas as pacientes receberam quimioterapia adjuvante, critério de elegibilidade neste estudo, entretanto, I I,4\% destas também receberam tratamento neoadjuvante. $O$ esquema quimioterápico contendo antracíclico foi usado em 56,1\% das pacientes. Foi possível, ainda, observar que as mulheres que utilizaram esquemas contendo antracíclicos apresentaram percentuais significativamente maiores $(p<0,05)$ de tumor acima de $2,0 \mathrm{~cm}(68,8 \%)$ e de linfonodos comprometidos (74,6\%), em relação às mulheres que utilizaram regimes com CMF (58,2\% e 40,8\%, respectivamente). O tratamento adjuvante hormonal foi utilizado em 52,8\% pacientes e o radioterápico em 76,4\%. Com relação às características relativas aos serviços de saúde, 293 mulheres (68,5\%) foram assistidas em serviços de saúde de natureza pública e $260(60,7 \%)$ não possuíam plano privado de saúde. Entre as pacientes assistidas no serviço de natureza privada, 81,5\% apresentaram intervalo entre diagnóstico e início do tratamento

\begin{tabular}{|c|c|c|}
\hline \multirow{3}{*}{ Variáveis } & \multirow{2}{*}{\multicolumn{2}{|c|}{ Casos }} \\
\hline & & \\
\hline & $\mathbf{N}$ & $\%$ \\
\hline \multicolumn{3}{|c|}{ Município de residência } \\
\hline Juiz de Fora & 196 & 45,8 \\
\hline Outras cidades & 232 & 54,2 \\
\hline \multicolumn{3}{|c|}{ Idade ao diagnóstico } \\
\hline $25-39$ anos & 64 & 15,0 \\
\hline $40-49$ anos & 136 & 31,8 \\
\hline $50-69$ anos & 202 & 47,2 \\
\hline$\geq 70$ anos & 26 & 6,1 \\
\hline \multicolumn{3}{|c|}{ Idade ao diagnóstico ( status menopausal) } \\
\hline Até 50 anos & 217 & 50,7 \\
\hline$>50$ anos & 211 & 49,3 \\
\hline \multicolumn{3}{|l|}{ Cor da pele $\#$} \\
\hline Branca & 299 & 69,9 \\
\hline Não-branca & 113 & 26,4 \\
\hline \multicolumn{3}{|c|}{ Tamanho do tumor" } \\
\hline$\leq 2 \mathrm{~cm}$ & 136 & 31,8 \\
\hline$>2 \mathrm{~cm}$ & 274 & 64,0 \\
\hline \multicolumn{3}{|c|}{ Linfonodos comprometidos ${ }^{\#}$} \\
\hline Não & 163 & 38,1 \\
\hline Sim & 256 & 59,8 \\
\hline \multicolumn{3}{|c|}{ Estadiamento ${ }^{\#}$} \\
\hline | & 51 & 11,9 \\
\hline$\|$ & 203 & 47,4 \\
\hline |II & 165 & 38,6 \\
\hline \multicolumn{3}{|c|}{ Tipo histológico } \\
\hline Ductal invasivo & 374 & 87,4 \\
\hline Lobularinvasivo & 27 & 6,3 \\
\hline Outrostipos & 27 & 6,3 \\
\hline \multicolumn{3}{|c|}{ Tempo entre diagnóstico e cirurgia } \\
\hline$\leq 12$ semanas & 362 & 84,6 \\
\hline$>12$ semanas & 65 & 15,2 \\
\hline \multicolumn{3}{|c|}{ Esquema de quimioterapia adjuvante ${ }^{\#}$} \\
\hline Antracíclico & 240 & 56,1 \\
\hline CMF & 184 & 43,0 \\
\hline \multicolumn{3}{|c|}{ Completude do esquema de quimioterapia adjuvante ${ }^{\#}$} \\
\hline Completa & 355 & 82,9 \\
\hline Incompleta & 60 & 14,0 \\
\hline \multicolumn{3}{|c|}{ Tempo entre diagnóstico e quimioterapia adjuvante ${ }^{\#}$} \\
\hline$\leq 12$ semanas & 265 & 61,9 \\
\hline$>12$ semanas & $|4|$ & 32,9 \\
\hline
\end{tabular}

"O total ( n) de cada variável difere devido à ocorrência de dados ignorados. 
CINTRA JRD ET AL.

quimioterápico de até 12 semanas, enquanto cerca de $40,3 \%$ dos casos assistidos no serviço público tiveram o início da quimioterapia após 12 semanas do diagnóstico $(p<0,000)$.

O tempo de sobrevida para todo o período de seguimento do estudo foi de 62,9 meses, com mediana de 66,0 meses (percentis $25 \%=60,0$ meses e $75 \%=76,5$ meses). Constatou-se que 24 mulheres $(5,6 \%)$ possuíam tempo de seguimento inferior a cinco anos e não foram a óbito, as quais representaram, portanto, os casos com seguimento incompleto. A função de sobrevida específica por câncer de mama para o período de cinco anos foi de 82\% (IC95\%: 78,0-85,4). Ao final do estudo, 8 I pacientes (18,9\%) morreram, sendo 73 (I7, | \%) por câncer de mama e o restante por causa não relacionada à doença. Entre os óbitos decorrentes do câncer de mama em cinco anos de seguimento $(n=73)$, observaram-se maiores percentuais de tumor maior que $2,0 \mathrm{~cm}$ em $76,1 \%$ dos casos $(n=54)$ e de linfonodos comprometidos em $87,5 \%$ dos casos $(n=63)$.

As principais funções de sobrevida não ajustadas para as variáveis do estudo são apresentada na Tabela 2. A sobrevida apresentou diminuição significativa de acordo com o aumento do tamanho tumoral (até 2,0cm: 87,2\%; maior que 2,0cm: 78,9\%) e do estadio da doença (I: 90\%; II: 89\%; III: 68,7\%). Em relação à faixa etária, não foi observada diferença significativa na sobrevida em relação a esta variável. No entanto, quando a idade foi dicotomizada para caracterizar o status menopausal ( $\leq 50$ anos e >50 anos), verificou-se uma melhor sobrevida para o grupo de mulheres com idade inferior a 50 anos $(p=0,02)$. Quando estratificada segundo o tipo de esquema quimioterápico, observou-se sobrevida superior para os casos que utilizaram esquemas com CMF (88\%; IC95\%: 82, I-92,0), em comparação com os que utilizaram regimes com antracílicos (77,6\%; IC95\%: $71,5-82,5)(p=0,008)$.

Apresentaram sobrevida desfavorável as mulheres que receberam ciclos incompletos de quimioterapia adjuvante $(71,7 \%)$, em relação àquelas que receberam ciclos completos $(83,5 \%)(p=0,03)$. Foram observadas melhores sobrevidas para as pacientes brancas (84\%), em relação às não-brancas (77\%), e para as mulheres que tiveram sua cirurgia realizada até 12 semanas após o diagnóstico (83,6\%), em comparação com aquelas com cirurgia realizada com mais de 12 semanas $(73,6 \%)$, sendo ambas as diferenças observadas na sobrevida próximas do valor de significância $(p=0,08)$. Não foi constatada diferença significativa na sobrevida em relação ao uso de radioterapia adjuvante. As mulheres que utilizaram hormonioterapia adjuvante apresentaram melhor sobrevida (85,3\%), em comparação com aquelas que não utilizaram tal terapêutica $(p=0,05)$. Na Figura I, são apresentadas as curvas de sobrevida estratificadas para as principais variáveis do estudo.

\section{Discussão}

No âmbito mundial, tem sido observado um aumento na incidência de câncer de mama, sendo que uma parte deste aumento, muito provavelmente, está relacionada ao crescente uso da mamografia por meio de programas de rastreamento dirigidos à população espećifica? Em vários páses desenvolvidos, a mortalidade por câncer de mama vem decrescendo, decréscimo este que tem sido atribuído à detecção

\begin{tabular}{lcc}
\hline & $\begin{array}{c}\text { Tabela 2 - Funções de sobrevida não } \\
\text { ajustadas para as variáveis do estudo }\end{array}$ & \\
\hline Variáveis & Sobrevida específica & p-valor** \\
& de 5 anos\% (IC95\%)* & \\
\hline
\end{tabular}

Idade ao diagnóstico ( $\approx$ status menopausal)

Até 50 anos

$86,2(80,7-90,2)$

$>50$ anos

$77,6(71,1-82,8)$

Cor da pele

Branca

$84,0(79,2-87,7)$

Não-branca

$77,0(68,0-83,9)$

Tamanho do tumor

$\leq 2 \mathrm{~cm}$

$87,2(80,2-91,9)$

$>2 \mathrm{~cm}$

$78,9(73,4-83,4)$

Linfonodos comprometidos

Não

$94,3(89,3-97,0)$

Sim

$73,6(67,5-78,8)$

$\mathbf{N}^{\circ}$ de linfonodos comprometidos

Nenhum

$94,3(89,3-97,0)$

I-3

$75,4(66,9-82,0)$

$4-9$

$74,6(61,8-83,6)$

$\geq 10$

$65,9(50,4-77,6)$

\section{Estadiamento}

Tempo entre diagnóstico e cirurgia

$\leq 12$ semanas

$83,6(79,3-87,1)$

$>12$ semanas

$73,6(60,1-83,2)$

Esquema de quimioterapia adjuvante

Antracílico

$77,6(71,5-82,5)$

CMF

$88,0(82,1-92,0)$

Completude do esquema de

quimioterapia adjuvante ${ }^{\#}$

$\begin{array}{ll}\text { Completa } & 83,5(79,1-87,1) \\ \text { Incompleta } & 71,7(57,5-81,9)\end{array}$

Uso de hormonioterapia adjuvante

Sim

Não

$85,3(79,9-89,4)$

$78,2(71,6-83,5)$

*IC95\%: intervalo de confiança de 95\%;

** Teste de Log-rank para cada variável. 
Figura I - Curvas de sobrevida estratificadas para as principais variáveis do estudo
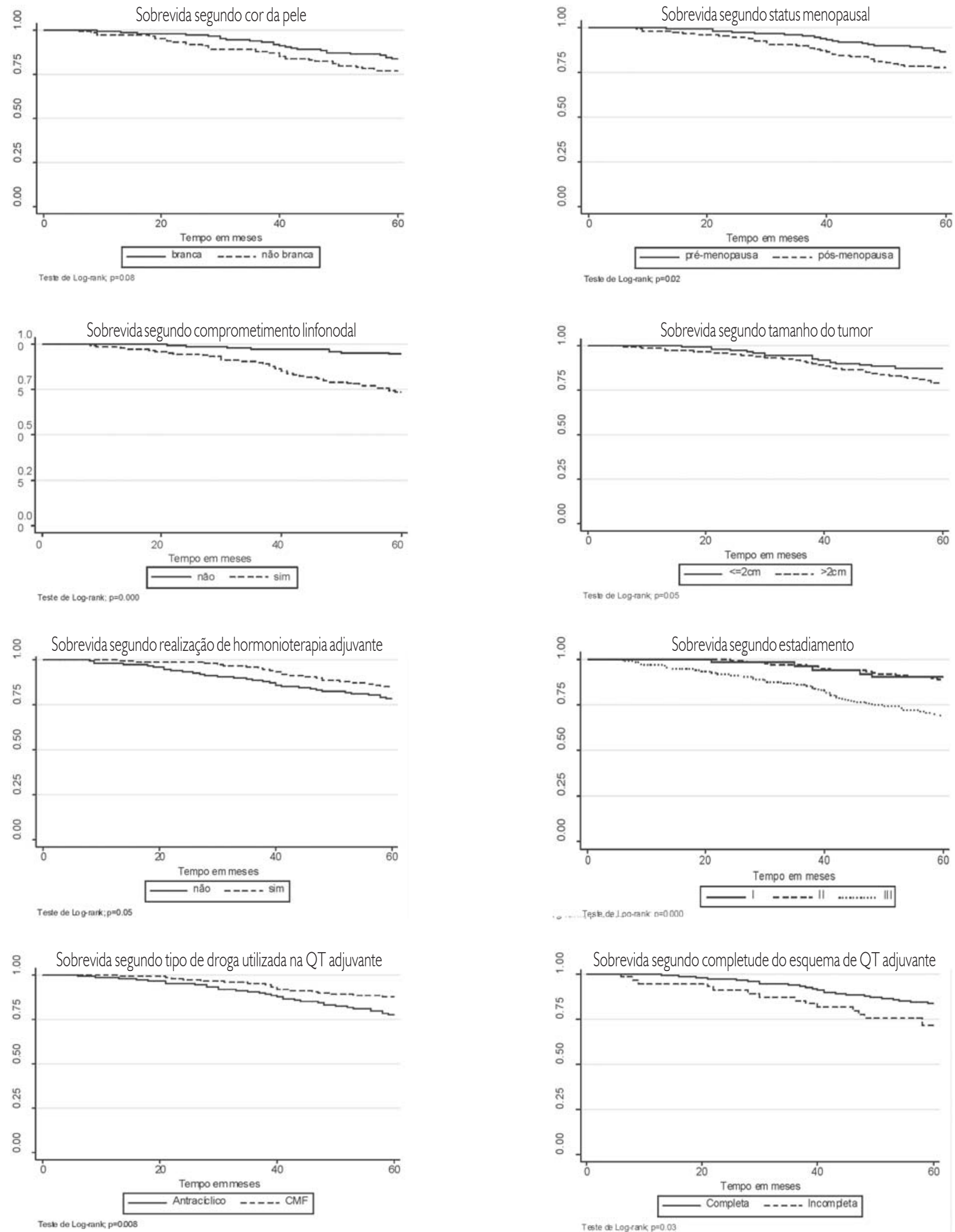
CINTRA JRD ET AL.

precoce, a qual possibilita a identificação da doença em fase mais inicial, e aos avanços do tratamento, 7, 16-19. Neste estudo, a sobrevida específica para o câncer de mama estimada em cinco anos foi de 80,2\%. Tal achado se apresenta próximo à sobrevida relativa de cinco anos observada na Europa (79\%) e inferior à observada nos Estados Unidos (89\%), ambas estimadas para casos incidentes recrutados entre 1990 e 1992, a partir dos respectivos registros de câncer de base populacional20. No que se refere ao Brasil, constatou-se que a sobrevida estimada neste estudo foi inferior à observada em apenas um dos estudos brasileiros analisados ${ }^{21-26}$. Entre estes estudos, destaca-se o conduzido por Mendonça et al. (2004) ${ }^{21}$, que analisaram dados de coorte hospitalar do Instituto Nacional do Câncer (INCA), Rio de Janeiro, verificando uma sobrevida geral em cinco anos de 75\%. As pacientes incluídas neste último estudo expressavam maiores semeIhanças com as do presente estudo, pois apresentavam diagnóstico de câncer de mama invasivo, efetuado entre 1995 e 1996, e tinham sido submetidas a tratamento cirúrgico. Finalmente, cabe ressaltar que, para uma adequada interpretação das mencionadas comparações de estimativas de sobrevida, devem ser considerados a população estudada, o período selecionado para os estudos, os critérios de elegibilidade, os métodos adotados para a análise e, principalmente, o fato de que todas as pacientes do estudo em tela foram submetidas a tratamento quimioterápico adjuvante, o que caracteriza uma população diferenciada no que se refere ao estadio da doença e ao acesso e as possibilidades de terapêutica.

A raça negra, em geral, está associada a um fator de pior prognóstico quando comparada a outros grupos racial-étnicos ${ }^{27,28}$, muito provavelmente em conseqüência do diagnóstico tardio da doença, do acesso mais dificultado à assistência terapêutica e de diferenças no tratamento e resultados ${ }^{28}$. Quando medidas de cuidado à saúde são instituídas tem sido verificada melhora na atenção desse grupo étnicoracial ${ }^{29,30}$. Field et al. (2005) ${ }^{27}$ verificaram, em estudo conduzido com pacientes com câncer de mama invasivo recrutados entre janeiro de 1993 a dezembro de 1998, que mulheres americanas brancas apresentaram melhor sobrevida que as afro-americanas, respectivamente de $81,6 \%$ e 73,8\%. Em nosso estudo, foi verificada sobrevida superior para as pacientes brancas (84\%) em relação às não-brancas (77\%), sendo tal diferença próxima do valor de significância $(p=0,08)$. A maioria das pacientes não-brancas $(77,8 \%)$ apresentava tamanho tumoral maior que dois centímetros $(p=0,004)$, o que pode estar associado à desigualdade de acesso aos serviços de saúde. Eisenberg $(2004)^{22}$ não encontrou diferenças estatísticas significativas nas curvas de sobrevida para a variável cor da pele. Uma grande presença de pardos e negros (42\%) foi verificada por De Moraes (2005) 23 na etapa de análise dos dados, o que pode ser justificada pela constituição racial da população baiana, entretanto não foi mencionado, nesse estudo, algum efeito desta variável sobre a sobrevida.

A idade média ao diagnóstico foi de $5 \mathrm{I}$, I anos, o que foi bastante semelhante a das pacientes estudadas por Abreu $(2002)^{31}$, que foi de 52,9 anos, e menor que a encontrada em outros estudos ${ }^{32,26,12}$, que foram respectivamente de 61 e 56,9 anos. Entretanto, em nossa análise, não se observou diferença significativa no tempo de sobrevida em relação a esta variável. Somente quando a idade foi dicotomizada para caracterizar o status menopausal ( $\leq 50$ anos e $>50$ anos) é que foi verificada melhor sobrevida para o grupo de mulheres com idade inferior a 50 anos $(p=0,02)$, o que pode ser explicado, em parte, pelo fato de o diagnóstico ser estabelecido mais precocemente para esse grupo de mulheres do que para os demais grupos etários, por pertencerem à faixa etária de maior incidência do câncer de mama, de acordo com os dados de literatura ${ }^{7}, 33$.

tipo histológico predominante foi o ductal infiltrante $(87,4 \%)$, achado semelhante aos outros estudos realizados $21,23,34,35,36$.

A presença de metástases para linfonodos axilares ainda é o principal fator prognóstico para o câncer de mama. $\bigcirc$ número de linfonodos comprometidos se constitui na mais relevante informação, certificado por vários estudos que demonstraram que a sobrevida das pacientes está diretamente relacionada ao número de linfonodos comprometidos $21,24,37$. Em nosso estudo, verificamos uma sobrevida inversamente proporcional ao número de linfonodos comprometidos. Para as pacientes com linfonodos negativos, a sobrevida específica em cinco anos foi de $94,3 \%$ e, para aquelas com comprometimento linfonodal maciço ( $\geq 10$ linfonodos positivos), a sobrevida foi de $65,9 \%$ $(p=0,000)$. Em relação ao estadiamento das mulheres no momento do diagnóstico, verificamos predominância dos estadios II e III (86\%), semelhante ao estudo de Pereira $(200 \mathrm{l})^{34}$, que foi de $83,2 \%$, podendo estar relacionada com a dificuldade de acesso dessas mulheres a exames que possibilitem o diagnóstico precoce.

No tocante à sobrevida segundo o tipo de esquema quimioterápico, pôde ser observada uma sobrevida superior para as mulheres que utilizaram esquemas com CMF (88\%), em comparação com a sobrevida observada para aquelas que utilizaram regimes com antracíclicos $(77,6 \%)(p=0,008)$. Cabe ressaltar que, nesta pesquisa, os esquemas com antracíclicos foram mais utilizados do que aqueles com CMF (56, I\% e 43\%, respectivamente), achado semelhante ao estudo de De Moraes (2005) 23. Entre as mulheres que utilizaram CMF, 70,6\% apresentavam estadio I e 65,0\% não apresentavam linfonodos comprometidos $(p=0000)$. Assim, a melhor sobrevida evidenciada nas mulheres que utilizaram o esquema com CMF pode ser explicada, em grande parte, por sua freqüente indicação para pacientes com tumores iniciais, sendo, portanto, a diferença observada mais relacionada com a gravidade da doença, que pode interferir na escolha do esquema quimioterápico, do que propriamente com o tipo de quimioterapia utilizada. Ressalte-se, ainda, que as pacientes que realizaram ciclos completos exibiram melhor sobrevida, em comparação com aquelas que receberam tratamento incompleto $(p=0,03)$. Para melhor compreensão desta diferença, deve ser levado em consideração o fato de que foram observados percentuais significativamente maiores de ciclos incompletos nas mulheres não-brancas do que as brancas (20\% e $12,1 \%$, respectivamente) e, ainda, nas mulheres na pós-menopausa do que naquelas na pré-menopausa (10,1\% e 18,1\%, respectivamente). Tais achados sinalizam para a influência do acesso diferencial aos cuidados de saúde, em decorrência de questões étnico-raciais ${ }^{27,28,29}$, e também para a menor aceitação/resposta (performance status) observada nas pacientes mais idosas ${ }^{38}$.

A utilização da hormonioterapia para o tratamento das pacientes foi percentualmente maior do que a não utilização. Verificou-se melhor sobrevida para as mulheres que receberam hormonioterapia adjuvante $(85,3 \%)$ em comparação com aquelas que não usaram $(78,2 \%)$ 
$(p=0,05)$. A adoção da terapia hormonal adjuvante tem uma elevada efetividade na melhoria da sobrevida das pacientes com câncer de mama ${ }^{39-44}$. Vale lembrar que, para esta população, a utilização de hormonioterapia não era orientada pelo resultado dos receptores hormonais para todos os casos, uma vez que tais exames não eram disponibilizados para a maioria das pacientes no período correspondente ao recrutamento dos casos.

A partir dos dados analisados, podemos concluir que as principais características que influenciaram positivamente a sobrevida específica em cinco anos para o câncer de mama na população de estudo foram: pré-menopausa $(p=0,02)$, raça branca $(p=0,08)$, tamanho do tumor $\leq 2,0 \mathrm{~cm}(p=0,05)$, ausência de comprometimento linfonodal $(p=0,000)$, estadios iniciais da doença $(\mid$ e $\|)(p=0,000)$, realização de ciclos completos de quimioterapia adjuvante $(p=0,03)$ e uso de hormonioterapia $(p=0,05)$. Assim, as piores sobrevidas foram verificadas para pacientes que apresentavam doença em estádio mais avançado.

A realização desta pesquisa reforça a importância de se trabalhar com as informações disponíveis nos serviços de saúde responsáveis pelo atendimento ao paciente com câncer no Brasil. Possibilitou a melhor caracterização do perfil e da sobrevida de pacientes com câncer de mama que utilizaram terapia quimioterápica adjuvante, em função da evolução/características da doença e da possibilidade de acesso à assistência. Assim, torna-se evidente a necessidade de ampliação, por parte dos órgãos competentes do setor de saúde local, do rastreamento do câncer de mama, especialmente para o grupo de mulheres considerado como de maior risco, bem como a garantia de tratamento oportuno para os casos diagnosticados, o que poderá influenciar positivamente o prognóstico da doença.

\section{Conflito de interesse: não há}

\section{SUMMARY}

Non-metastatic breast CANCER Specific-surVival OF PATIENTS AFTER TREATMENT WITH ADJUVANT CHEMOTHERAPY

OBJECTIVE. Analyze the 5-year breast cancer specific-survival rate of women diagnosed with invasive non-metastatic disease, who as part of their primary treatment underwent surgery followed by adjuvant chemotherapy.

METHODS. Four hundred twenty eight patients diagnosed between 1998 and 2000 were recruited from all oncology services of the municipality of Juiz de Fora, MG, Brasil. Survival time was counted from the date of the histopathological diagnosis and the date of death due to breast cancer was considered the adverse event. Women alive until December 2005, the final date of the follow-up, were censored. For those who interrupt treatment, censor date was the lastfollow-up in the medical records. Kaplan-Meier survival curves were estimated, with the differences assessed by the log-ranktest. Results: Mean age was 51.2 years, and most (72.6\%) were Caucasian. Clinical Stages II (47.4\%) and III (38.6\%) predominated. Breast cancer specific five-year survival rate was $82.0 \%$. A worstsurvival was observedamong women with disease diagnostic before menopause $(p=0.02)$, with tumor size greater than $2.0 \mathrm{~cm}(p=0.05)$, with lymph node involvement $(p=0,000)$, in a more advanced disease stage $(p=0.000)$, on a full adjuvant chemotherapy regimen $(p=0.03)$, and who used hormone therapy $(p=0.05)$.

CONCLUSION. This research allowed identification of the profile and disease survival of breast cancer patients who used adjuvant chemotherapy. These results stimulated the adoption of intensive strategies by the local health authorities for disease control and prevention in this population, emphasizing the increasing need of breast cancer screening, mainly for women considered as of high risk and the availability of timely treatment for all cases diagnosed. [Rev Assoc Med Bras 2008; 54(4): 339-46]

KEY WORDS: Breast cancer. Chemotherapy. Prognosis. Survival analysis.

\section{REFERÊNCIAS}

I. Bray F, McCarron P, Parkin DM. The changing global patterns of female breast cancer incidence and mortality. Breast Cancer Res. 2004;6(6):229-39.

2. McPherson K, Steel CM, Dixon JM. ABC of breast diseases. Breast cancer: epidemiology, risk factors and genetics. BMJ. 2000;32I (726I):624-8.

3. Parkin DM, Bray F, Ferlay J, Pisani P. Global cancer statistics, 2002. CA Cancer J Clin. 2005;55(2):74- 08 .

4. Guerra MR, Gallo CVM, Mendonça GAS. Risco de câncer no Brasil: tendências e estudos epidemiológicos mais recentes. Rev Bras Cancerol. 2005;5I (3):227-34.

5. Osborne CK, Clark GM, Ravdin PM. Adjuvant systemic therapy of primary breast cancer. In: Harris JR, Morrow M, Lippman ME, Hellman S. Diseases of the breast. $2^{\text {nd }}$ ed. New York: Lippincott-Raven Publishers; 1996. p.548-78.

6. Parkin DM, Whelan SL, Ferlay J, et al., editors. Cancer incidence in five continents. Lyon: International Agency for Research on Cancer; 2002. (v.8, IARC Scientific Publications N. I 55)

7. International Agency for Research on Cancer. Globocan 2002. [cited 2008 jan 9]. Available from: http://www-dep.iarc.fr/.

8. Ministério da Saúde. Instituto Nacional do Câncer (INCA). Estimativa 2006: incidência de câncer no Brasil [citado 20 jun 2007]. Disponível em: http://www.inca.gov.br/estimativa/2006/.

9. Smigal C, Jemal A, Ward E, Cokkinides V, Smith R, Howe HL, etal. Trends in breast cancer by race and ethnicity: update 2006. CA Cancer J Clin. 2006;56(3): 168-83.

I0. National Cancer Institute. 2004. [citado 2007 dez 06]. Available from: http://www.cancer.gov/cancerinfo/pdq/treatment/breast/ HealthProfessional\#Section27.

I।. Ministério da Saúde. Instituto Nacional do Câncer (INCA). Registros Hospitalares de Câncer (RHC) - 1998. Instituto Nacional do Câncer. [citado 2006 dez 14]. Disponível em: http://www.inca.gov.br.

12. Guerra MR. Sobrevida e fatores prognósticos para o câncer de mama em Juiz de Fora, Minas Gerais, na coorte diagnosticada entre 1998 e 2000 [tese]. Rio de Janeiro: Instituto de Medicina Social, Universidade do Estado do Rio de Janeiro; 2007.

13. TNM: classificação de tumores malignos. $6^{a}$ ed. Rio de Janeiro: INCA; 2004.

14. Anderson WF, Jatoi I, Devesa SS. Distinct breast cancer incidence and prognostic patterns in the NCl's SEER program: suggesting a possible link between etiology and outcome. Breast Cancer Res Treat. 2005;90(2): 127-37.

15. Armitage P, Berry G. Survival analysis. In: Armitage P, Berry G. Statistical methods in medical research. $2^{\text {nd }}$ ed. Oxford: Oxford Scientific Publications; 1987.

16. Matti SA. Adjuvant therapy of primary breast cancer: a review of key findings from the 7th International Conference, St. Gallen, February $200 \mathrm{I}$. Oncologist. 2001;6(4):376-85.

17. Parkin DM, Bray F, Ferlay J, Pisani P. Global cancer statistics, 2002. Ca Cancer J Clin. 2005;55(I):74- 108. 
CINTRA JRD ET AL.

18. Urbain JL. Breast cancer screening, diagnostic accuracy and health care polities. J Ass Med Canad. 2005; I72(2):2 I0-I.

19. Jemal A, Siegel R, Ward E, Murray T, Xu J, Thun MJ. Cancer statistics, 2007. CA Cancer J Clin. 2007;5( I):43-66.

20. Sant M, Allemani C, Berrino F, Coleman MP, Aareleid T, Chaplain, G, et al. Breast carcinoma survival in Europe and the United States. Cancer. 2004; I00(4):7I 5-22.

21. Mendonça GAS, Silva AM, Caula WM. Características tumorais e sobrevida de cinco anos em pacientes com câncer de mama admitidas no Instituto Nacional de Câncer, Rio de Janeiro, Brasil. Cad Saúde Pública. 2004;20(5): I 232-9.

22. Eisenberg ALA. Sobrevida de cinco anos para pacientes com carcinoma ductal infiltrante de mama sem comprometimento de linfonodos axilares. Coorte hospitalar, 1992-1996, Rio de Janeiro, Brasil [tese] Rio de Janeiro: Escola Nacional de Saúde Pública, Fundação Oswaldo Cruz; 2004

23. De Moraes ED, Alban LBV, Costa R, et al. Epidemiologia, características tumorais e sobrevida global de 1565 pacientes com câncer de mama em acompanhamento no Núcleo de Oncologia da Bahia, Brasil. Rev Soc Bras Cancerol. 2005; 29(I):4- 12 .

24. De Abreu E. Câncer de mama feminina em Goiânia: análise da sobrevida em I0 anos na coorte diagnosticada entre 1988-90 [tese]. Rio de Janeiro: Escola Nacional de Saúde Pública, Fundação Oswaldo Cruz; 2002

25. Pereira WMM. Mortalidade e sobrevida por câncer de mama, no estado do Pará. [dissertação]. Rio de Janeiro: Escola Nacional de Saúde Pública, Fundação Oswaldo Cruz; 200 I

26. De Moraes AB, Zanini RR, Turchiello MS. Estudo da sobrevida de pacientes com câncer de mama atendidas no hospital da Universidade Federal de Santa Maria, Rio Grande do Sul, Brasil. Cad Saúde Pública. 2006:22(10): 2219-28.

27. Field TS, Buist DSM, Doubeni C, Enger S, Fouaysi H, Hart G, et al. Disparities and survival among breast cancer patients. J Natl Cancer Inst Monogr. 2005;35(I):88-95.

28. Hsu JL, Glaser SL, West DW. Racial/ethnic differences in breast cancer survival among San Francisco Bay area women. I Natl Cancet Inst. 1997;89(17): | 31 |-2

29. Micheli A, Gatta G, Verdecchia A. Studying survival of cancer patients in different in different populations: its potential and role. Tumori. 1997;83(I):3-8.

30. Ruger JPM. Measuring disparities in health care. BM]. 2006;333(7562):274.

31. De Abreu E. Câncer de mama feminina em Goiânia: análise da sobrevida em 10 anos na coorte diagnosticada entre 1988-90 [tese]. Rio de Janeiro: Escola Nacional de Saúde Pública, Fundação Oswaldo Cruz: 2002.

32. Simon MS, Banerjee M, Crossley-May H, Vigneau FD, Noone AM, Schwartz Z, et al. Racial differences in breast cancer survival in Detroit metropolitan area. Breast Cancer Res Treat. 2006;97(2): | 49-55.

33. American Cancer Society. Cancer: facts \& figures [cited 2007 jun I0]. Available from: http://www.cancer.org/docroot/PRO/content/ PRO_I_I_Cancer_Statistics_2006_presentation.asp.
34. Pereira WMM. Mortalidade e sobrevida por câncer de mama, no estado do Pará. [dissertação]. Rio de Janeiro: Escola Nacional de Saúde Pública, Fundação Oswaldo Cruz; 2001.

35. Brito C, Portela MC, De Vasconcelos MTL. Assistência oncológica pelo SUS a mulheres com câncer de mama no Estado do Rio de Janeiro. Rev Saúde Pública. 2005;39(6):874-8I

36. De Moraes AB, Zanini RR, Turchiello MS. Estudo da sobrevida de pacientes com câncer de mama atendidas no hospital da Universidade Federal de Santa Maria, Rio Grande do Sul, Brasil. Cad Saúde Pública. 2006:22(10):2219-28.

37. Lin Y-C, Chen S-C, Chang H-K. Identifying good prognosis group of breast cancer patients with I-3 positive axillary nodes for adjuvant cyclophosphamide, methotrexate and 5-fluorouracil (CMF) chemotherapy. Jpn J Clin Oncol. 2005;35(9):5 I4-9.

38. Repetto BL, Fratino L, Audisio RA, Venturino A, Gianni V, Vercelli M, et al. Comprehensive Geriatric Assessment Adds Information to Eastern Cooperative Oncology Group Performance Status in Elderly Cancer Patients: an Italian Group for Geriatric Oncology Study. J Clin Oncol. 2002;20(2):494-502.

39. Schrag D, Kuntz KM, Garber JE, Weeks JC, Redmond CK, Kavanah M, Cronin WM, et al. Effects of prophylactic mastectomy and oophorectomy on life expectancy women with BRCA I or BRCA2 mutations. N Engl J Med. 1997;336(20): | 465-7|.

40. Fisher B, Constantino JP, Wickerham DL. Tamoxifen for the prevention of breast cancer: report of the National Surgical Adjuvant Breast and Bowel Project P-I Study. J Natl Cancer Inst. 1998;90( I 8): I 37 | -88.

4I. Offit K, Kauff N, Satagopan J, et al. Risk-reducing salpingo-oophorectomy in women with BRCAI and BRCA2 mutations. In: American Society of Clinical Oncology. Thirty-Eighth Annual Meeting 2002, Orlando. Proc Am Soc Clin Oncol. 2002;2l (abstr 3).

42. Baum M, Budzar AU, Cuzick J, Forbes J. Houghton JH, Klijn JG, et al. The Atac Trialists'group. Anastrozole alone or in combination with tamoxifen versus tamoxifen alone for adjuvant treatment of postmenopausal women with early breast cancer: first results of the ATAC randomised trial. Lancet. 2002;359(9324):2131-9.

43. Baum M, Buzdar A, Cuzick J, Forbes 」, Houghton 」, Howell A, et al. The Atac Trialists'group. Anastrozole alone or in combination with tamoxifen alone for adjuvant treatment of postmenopausal women with early-stage breast cancer. Result of the ATAC (Arimidex ${ }^{\circledR}$, tamoxifen, alone or in combination) trial efficacy and safety update analysis. Cancer. 2003:98(9): | 802- 10.

44. Howell A, Cuzick J, Baum M, Buzdar AU, Dowsett M, Forbes JF, et al. The Atac Trialists' GrouP. Results of the ATAC (Arimidex ${ }^{\circledR}$, Tamoxifen alone or in combination) trial after completion of 5 years adjuvant treatment for breast cancer. Lancet. 2005:365(9453):60-2.

Artigo recebido: 07/09/07

Aceito para publicação: 30/01/08 